\title{
Verification of Carbon Density Profile Measurements with Charge Exchange Spectroscopy Using Hydrogen and Deuterium Neutral Beams
}

\author{
Katsumi IDA $^{1,2)}$, Mikirou YOSHINUMA ${ }^{1,2)}$, Tatsuya KOBAYASHI ${ }^{1,2)}$, Yutaka FUJIWARA ${ }^{1)}$, \\ Jun $\mathrm{CHEN}^{3)}$, Izumi MURAKAMI ${ }^{1,2)}$, Masashi KISAKI ${ }^{1,2)}$, Masaki OSAKABE ${ }^{1,2)}$ \\ and LHD Experiment Group ${ }^{1)}$ \\ 1) National Institute for Fusion Science, National Institutes of Natural Sciences, Toki, Gifu 509-5292, Japan \\ ${ }^{2)}$ SOKENDAI (The Graduate University for Advanced Studies), Toki, Gifu 509-5292, Japan \\ ${ }^{3)}$ School of Nuclear Science and Technology, University of Science and Technology of China, Hefei 230026, China
}

(Received 6 February 2019 / Accepted 23 March 2019)

\begin{abstract}
Verification of carbon density profile measurements with charge exchange spectroscopy of CVI $(\Delta n=8-7$, $\lambda=529.2 \mathrm{~nm}$ ) using hydrogen and deuterium neutral beams is described. This is a key issue in the study of the comparison of the carbon density profile between hydrogen and deuterium plasma, because the hydrogen neutral beams are injected in the hydrogen plasma and the deuterium neutral beams are injected in the deuterium plasma. By applying the precise beam attenuation calculation using precise atomic data, identical carbon density profile is obtained for the plasma with hydrogen beam and deuterium beam. A Verification of the measurement is performed by comparison of carbon density profile measured with charge exchange spectroscopy and increment of electron density profile just after the carbon pellet injection.
\end{abstract}

(c) 2019 The Japan Society of Plasma Science and Nuclear Fusion Research

Keywords: charge exchange spectroscopy, carbon density profile, helical plasma

DOI: $10.1585 /$ pfr.14.1402079

\section{Introduction}

Isotope effect on the plasma confinement is an important issue in toroidal plasma. Recent gyrokinetic simulation demonstrates that the impurity density gradient has a significant impact of the stabilization/destabilization of the ion-temperature gradient (ITG) mode [1-3]. ITG mode is predicted to be stabilized when the impurity density gradient becomes negative with peaked density profile, while it is destabilized when the impurity density gradient becomes positive with hollow density profile. Therefore, it is important to measure the radial profile of impurity density precisely enough to investigate the correlation between impurity gradient and heat transport both in hydrogen and deuterium core plasma, where some isotope effect on heat transport is observed [4]. The comparison of impurity density profile between hydrogen and deuterium plasma has become highlighted because the significant impact of impurity density gradient on turbulence and heat transport has been recognized recently $[5,6]$. Typically, the hydrogen beam is injected in the hydrogen plasma and the deuterium beam is injected in the deuterium plasma. Charge exchange spectroscopy has been used to measure the precise impurity density profile as well as the ion temperature and the plasma flow velocity profiles [7-10]. In this technique, the impurity density is evaluated from the ra- dial profile of intensity of charge exchange line and beam density profile derived with beam attenuation calculation.

The stopping cross section is larger for the deuterium beam than for the hydrogen beam because of the lower velocity of deuterium beam. Due to the stronger beam attenuation of the deuterium beam, the intensity of the charge exchange line decreases more sharply for the deuterium beam than for the hydrogen beam. In contrast, the emission cross section is smaller for the deuterium beam than the hydrogen beam. Then the intensity of the charge exchange line becomes weaker for the same beam power. The difference in beam velocity between hydrogen and deuterium beam has a significant impact on the evaluation of beam attenuation and intensity of the charge exchange line. Therefore, the verification of carbon density profile measurements using charge exchange spectroscopy is a fundamental issue in the study of comparison of impurity density profile between hydrogen and deuterium plasma.

In this paper, the verification of carbon density profile measurements performed in LHD is described. Atomic data (stopping cross section and emission cross section) is mentioned in Sec. 3. A new precise beam attenuation calculation technique is discussed in Sec. 4. In Sec. 5, the carbon density profile measurements for hydrogen and deuterium beam for the similar condition and the verification using the carbon pellet injection are discussed. 


\section{Determination of Impurity Density Profile by Charge Exchange Spec- troscopy}

In general, both beam profile and attenuation by the plasma are calculated simultaneously for the beam from each hole of the ion source with finite divergence, and with the precise beam attenuation calculation. Although the beam attenuation depends on the density profiles and changes in time, beam profile is virtually unchanged for the optimized neutral beam operation. Therefore, the calculation time can be shortened significantly by separating the calculation of beam profile which is typically unchanged and the calculation of the attenuation which depends on the plasma density. In order to separate these two calculations, the vector from the observation point to the center of the ion source (beam line vector: $\vec{l}\left(d, R_{i}, z\right)$ ) is introduced. Here, $d, R_{i}$, and $z$ are the distance from the beam line, major radius and height of the intersection between beam line and the line of sight. In this technique, the beam density without attenuation and beam line vector is calculated in advance, because it is not dependent on the plasma density. The beam density with attenuation is calculated backward (from observation point towards the ion source) for each time slice of Thomson scattering by integrating the electron density along the beam line vector using the density measured. The beam attenuation calculation time with this technique is considerably shorter than the conventional beam attenuation calculation, where the beam density is calculated forward (from ion source to the observation point).

Figure 1 shows the schematics of the experimental setup of impurity density measurements with charge exchange spectroscopy in LHD. The neutral beam to excite the charge exchange line of carbon CVI is perpendicular to the magnetic flux surface along major radius, while the line of sight of charge exchange spectroscopy is tangential and nearly parallel to magnetic flux surface at the mid-plane of the plasma $(z=0)$. There are two beam lines (beam line A and beam line B) and each beam line has two ion source (upper and lower ion source). There are also two viewing arrays. One is for low field side of the plasma (from port $7 \mathrm{~T}$ ) and the other is for high field side of the plasma (from port 6O). These two viewing arrays have an overlapping region near the plasma center $\left(R_{i}=3.8 \mathrm{~m}\right)$ to make a relative calibration between the two viewing arrays.

The intensity of impurity density measured with charge exchange spectroscopy $I\left(R_{i}, z\right)$ is given by

$$
I\left(R_{i}, z\right)=\frac{\Omega}{4 \pi} n_{z} \sum_{j=1}^{3}\langle\sigma v\rangle_{\lambda}^{j} \int n_{b}^{j}\left(d, R_{i}, z\right) d s .
$$

Here, $n_{b}^{j}\left(d, R_{i}, z\right)$ is beam density and $d, R_{i}$, and $z$ are distance from the beam line, major radius, and height of the intersection between beam line and line of sight. $\langle\sigma v\rangle_{\lambda}^{j}$ is the emission cross section for the transition of $\Delta n=8-7$

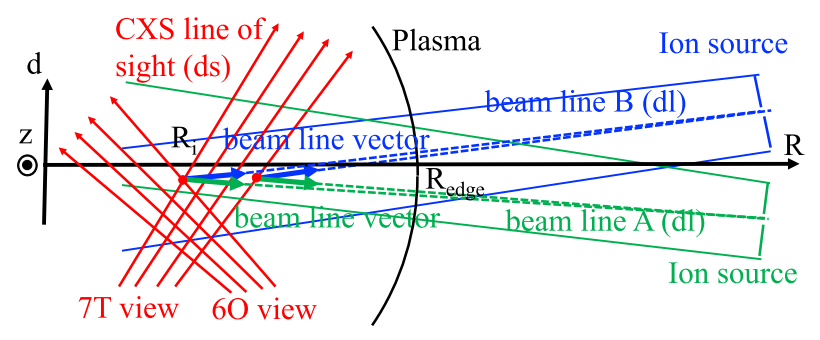

Fig. 1 Schematics of experimental setup of impurity density measurements with charge exchange spectroscopy in LHD.

of CVI $(\lambda=529.2 \mathrm{~nm}) . E_{b}^{j}$ is the beam energy per atomic unit ( 1 for hydrogen beam and 2 for deuterium beam) for $j$-th components of beam (full, half, and one-third energy, $\left.E_{b}^{1}=2 E_{b}^{2}=3 E_{b}^{3}\right)$. $\Omega$ is the solid angle of the optics of charge exchange spectroscopy. $n_{z}$ is impurity density averaged within the beam width along the line of sight of $d s$. In the toroidal view where the line of sight is nearly parallel to the magnetic flux surface, the impurity density is almost constant within the beam width. Therefore, the difference between local impurity density and $n_{z}$ is negligible.

Beam density $n_{b}^{j}\left(d, R_{i}, z\right)$ is obtained by the beam attenuation calculation along beam line vector of $\vec{l}\left(d, R_{i}, z\right)$ plotted in Fig. 1 as

$$
n_{b}^{j}\left(d, R_{i}, z\right)=n_{b 0}^{j}\left(d, R_{i}, z\right) \exp \left[-\int_{R_{i}}^{R_{\text {edge }}} \sigma_{s} n_{e} d l\right]
$$

Here $n_{b 0}^{j}\left(d, R_{i}, z\right)$ is the beam density without attenuation by plasma (indicated as vacuum) for $j$-th component of the neutral beam. $\sigma_{s}$ is a stopping cross section of the neutral beam due to plasma. New technique for precise beam attenuation calculation is applied. The pair of beam density without attenuation and beam line vector, $n_{b 0}^{j}\left(d, R_{i}, z\right)$ and $\vec{l}\left(d, R_{i}, z\right)$, are calculated in advance. The beam density, $n_{b}^{j}\left(d, R_{i}, z\right)$, has exponential decay inside the plasma determined by the stopping cross section and line integrated density along the beam line vector of $\vec{l}\left(d, R_{i}, z\right)$ from the measurement point of $R_{i}$ to the plasma edge $\left(R_{\text {edge }}\right)$.

$$
n_{b 0}^{j}\left(w, R_{i}, z\right)=F\left(w, R_{i}, z\right)\left(f_{j} P / E_{b}^{j}\right) / v_{j} .
$$

$f_{j}$ is power fraction of the $j$-th component of the neutral beam and $v_{j}, E_{b}^{j}$, and $\mathrm{P}$ are beam velocity, beam energy, and total power of the neutral beam.

\section{Atomic Data for the Carbon Den- sity Profile Measurements}

In this section, the stopping cross section for the beam and emission cross section for charge exchange reaction between impurity and neutral beam is described for the energy range of hydrogen and deuterium beam. In general, Atomic Data and Analysis Structure (ADAS) [11] is 
commonly used to calculate the stopping cross section for the beam and emission cross section for each time slice. This is explained by the fact that the stopping cross section for the beam and emission cross section depends on the plasma parameters such as impurity concentration and the density and temperature in addition to the beam energy. In this study, the parameter dependence is extracted from the ADAS and the stopping cross section for the beam and emission cross section expressed with a function of the beam energy and multiple correction coefficients.

The density and temperature dependence of the stopping cross section is quite weak compared with the energy dependence of the neutral beam. The stopping cross increases, as the impurity concentration $\left(n_{z} / n_{e}\right)$ is increased. However, the beam energy dependence of this effect is relatively weak. The density and temperature dependence of the stopping cross section is very weak compared with energy dependence of the neutral beam. The stopping cross increases, as the impurity concentration $\left(n_{z} / n_{e}\right)$ is increased. However, the beam energy dependence of this effect is relatively weak. Therefore, the impurity concentration, electron density, and temperature dependences are extracted as correction factors of $\xi_{z}, \xi_{n e}$, and $\xi_{T e}$ and the stopping cross section can be approximately expressed as $\sigma_{s}\left(E_{b}^{j}, n_{z}, n_{e}, T_{e}\right)=\xi_{z}\left(n_{z} / n_{e}\right) \times \xi_{n e}\left(n_{e}\right) \times \xi_{T e}\left(T_{e}\right) \times \sigma\left(E_{b}^{j}\right)$.

Figure 2 shows the stopping cross section of neutral beam as a function of beam energy per atomic unit and correction factors due to impurity concentration, electron density, and electron temperature. Because the stopping cross section increases towards the lower beam energy per atomic unit, the stopping cross section in the deuterium (D) beam is larger than that of hydrogen $(\mathrm{H})$ beam. The beam energy per atomic unit for the full energy component $(j=1)$ is $30-48 \mathrm{keV} / \mathrm{amu}$ for $\mathrm{H}$ - beam and 19 $30 \mathrm{keV} / \mathrm{amu}$ for D-beam. The precise determination of the stopping cross section is important for evaluating the carbon density profile because the slight change in the stopping cross section has a larger impact on beam density profile when the plasma density is high. Here, $\sigma\left(E_{b}^{j}\right)$ is the stopping cross section for the pure plasma without impurity and for the fixed electron density and temperature at $Z_{\mathrm{eff}}=1, n_{e}=1 \times 10^{19} \mathrm{~m}^{-3}$, and $T_{e}=2 \mathrm{keV}$. The correction factors are defined as the ratio of stopping cross section to that for the reference parameters of $E_{b}=40 \mathrm{keV}$, $Z_{\mathrm{eff}}=1, n_{e}=1 \times 10^{19} \mathrm{~m}^{-3}$, and $T_{e}=2 \mathrm{keV}$. The enhancement of stopping factor by impurity depends on the impurity species. The correction factor is larger for the impurity with larger charge as seen in Fig. 2. The emission cross section also depends on the beam energy, $Z_{\mathrm{eff}}$, ion density and temperature due to the $l$-mixing by ion-ion collisions. The emission cross section $\langle\sigma v\rangle_{\lambda}^{j}\left(E_{b}^{j}, Z_{\mathrm{eff}}, n_{i}, T_{i}\right)=$ $\zeta_{z}\left(Z_{\text {eff }}\right) \times \zeta_{n i}\left(n_{i}\right) \times \zeta_{T i}\left(T_{i}\right) \times Q\left(E_{b}^{j}\right)$. Figure 3 shows the emission cross section of CVI $(\Delta n=8-7, \lambda=529.2 \mathrm{~nm})$ as a function of beam energy per atomic unit for $Z_{\mathrm{eff}}=2$, $n_{e}=5 \times 10^{19} \mathrm{~m}^{-3}, T_{e}=2 \mathrm{keV}$ and correction factors due to
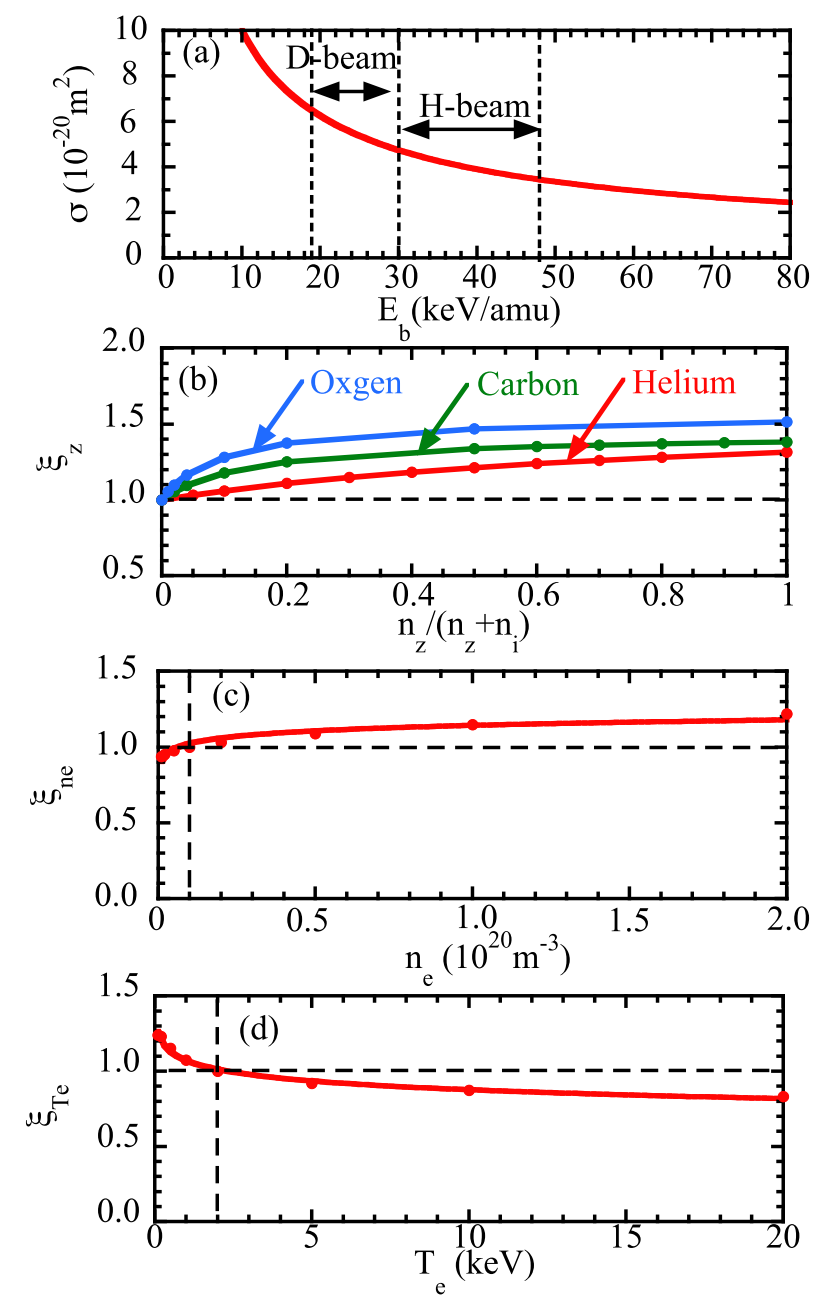

Fig. 2 (a) Stopping cross section of neutral beam as a function of beam energy per atomic unit for $Z_{\mathrm{eff}}=1, n_{e}=$ $1 \times 10^{19} \mathrm{~m}^{-3}$, and $T_{e}=2 \mathrm{keV}$ and correction factors due to (b) impurity concentration, (c) electron density, and (d) electron temperature. The beam energy range for hydrogen $(\mathrm{H})$ beam and deuterium $(\mathrm{D})$ beam are indicated.

$Z_{\text {eff }}$, electron density, and ion temperature. The correction factors are defined as the ratio of stopping cross section to that for the reference parameters of $E_{b}=40 \mathrm{keV}, Z_{\mathrm{eff}}=2$, $n_{e}=5 \times 10^{19} \mathrm{~m}^{-3}$, and $T_{i}=2 \mathrm{keV}$.

Although the emission cross section between fully ionized carbon and hydrogen or deuterium atom with an electron in the ground state of $n=1$ decreases sharply at lower beam energy below $20 \mathrm{keV}$, the emission cross section between fully ionized carbon and hydrogen or deuterium atom with a electron in the meta-stable state of $n=2$ increases as the beam energy is decreased [12]. Because the emission cross section for the hydrogen and deuterium atom with an electron in the meta-stable is relatively large, only the small fraction of the population in the metastable can change the emission cross section curve significantly. The fraction of the population in the meta-stable, $P_{n=2} / P_{n=2}$, is experimentally determined to be $2 \%$ from the precise measurements of the apparent Doppler shift of car- 

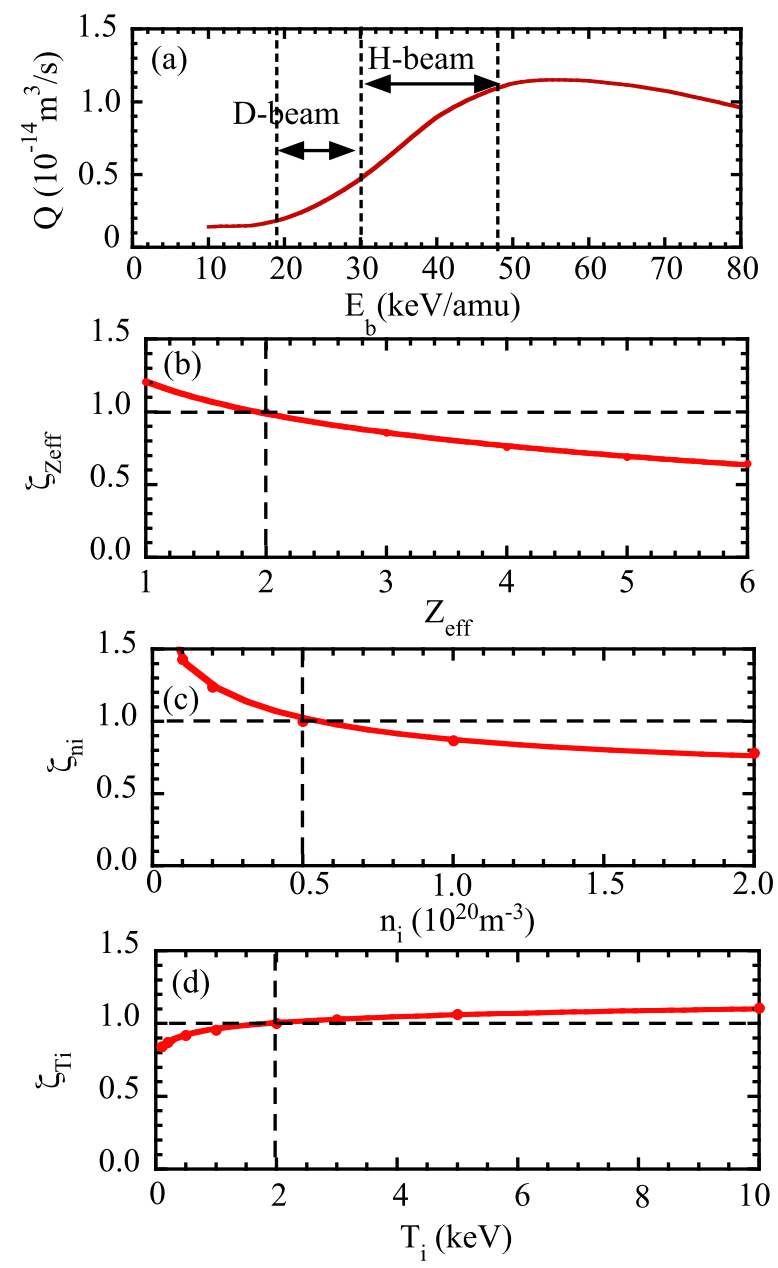

Fig. 3 (a) Emission cross section of CVI $(\Delta n=8-7, \lambda=$ $529.2 \mathrm{~nm}$ ) as a function of beam energy per atomic unit for $Z_{\mathrm{eff}}=2, n_{e}=5 \times 10^{19} \mathrm{~m}^{-3}, T_{e}=2 \mathrm{keV}$, and metastable population of $P_{n=2} / P_{n=1}=0.2 \%$ and correction factors due to (b) $Z_{\mathrm{eff}}$, (c) electron density, and (d) ion temperature. The beam energy range for hydrogen $(\mathrm{H})$ beam and deuterium (D) beam are indicated.

bon ion due to the energy dependent cross section effect in LHD [13]. The magnitude of the emission cross section below $20 \mathrm{keV}$ becomes important to evaluate the contribution from the charge exchange reaction between fully ionized carbon and one-half $\left(E_{b}^{1}=20 \mathrm{keV} / \mathrm{anu}\right)$ and onethird energy ( $\left.E_{b}^{1}=13.3 \mathrm{keV} / \mathrm{anu}\right)$ component of hydrogen beam and a half $\left(E_{b}^{1}=10 \mathrm{keV} / \mathrm{anu}\right)$ and one-third energy $\left(E_{b}^{1}=20 \mathrm{keV} / \mathrm{anu}\right)$ component of deuterium beam at nominal operation operation $\left(E_{b}^{1}=40 \mathrm{keV} / \mathrm{amu}\right.$ for H-beam and $E_{b}^{1}=30 \mathrm{keV} / \mathrm{anu}$ for D-beam).

\section{Beam Attenuation Calculation}

In order to calculate the beam density precisely, estimate of the beam profile at the intersection between the beam line and the line of sight is important. This is because the beam density usually decreases due to the finite beam divergence angle and steering angle even without the atten-

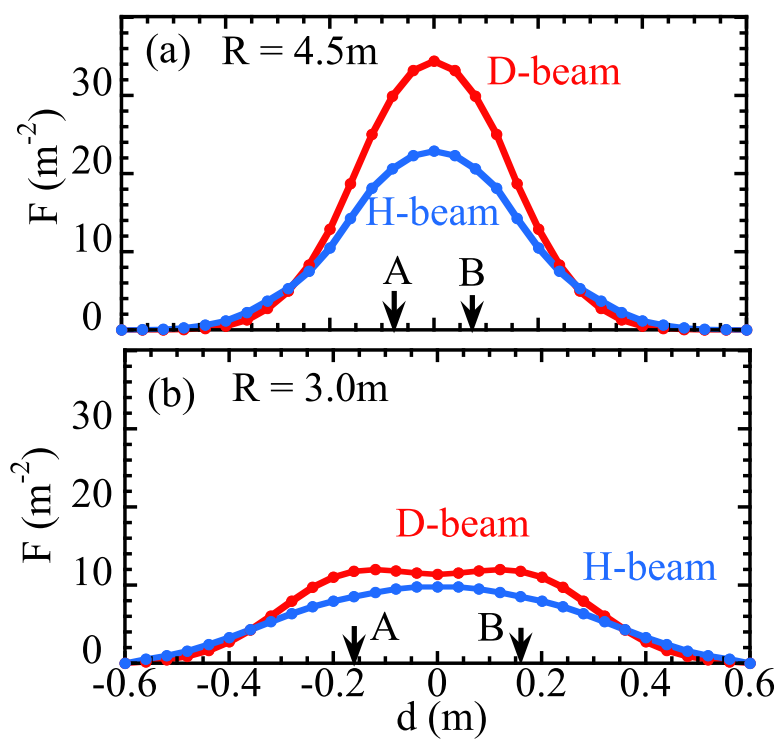

Fig. 4 Beam probability density function, $F$, in vacuum at the mid-plane $(z=0)$ as a function of beam horizontal distance at the major radius of (a) $4.5 \mathrm{~m}$ and (b) $3.0 \mathrm{~m}$.

uation by plasma. The beam probability distribution function, $\mathrm{F}$, is evaluated by the beam simulation code, where the trajectory of neutral beam emitted from each hole of the ion source with the beam divergence angle measured is calculated.

Figure 4 shows the beam probability density function, $F$, in vacuum as a function of the perpendicular distance from the beam line at the major radius near the plasma edge at the low magnetic field side $(R=4.5 \mathrm{~m})$ and near the plasma edge at the high magnetic field side $(R=3.0 \mathrm{~m})$. The arrows indicate the position of the beam line A and the beam line B. The distance between the beam line A and the beam line B is $15 \mathrm{~cm}$ at $R=4.5 \mathrm{~m}$ and $30 \mathrm{~cm}$ at $R=3.0 \mathrm{~m}$ This is because the pivot point of the neutral beam of four ion sources locates near the injection port of LHD ( $R=4.9 \mathrm{~m}$ of up and down ion sources and $R=$ $5.72 \mathrm{~m}$ of left (A) and right (B) ion sources). Owing to the beam steering and divergence, the beam density at the center of two beam lines $(d=0)$ decreases and the beam probability distribution function becomes wider. There are distinct differences in the beam profiles between H-beam and D-beam. The beam profile of D-beam is narrower than that of H-beam, The beam density at the center of two Dbeam lines $(d=0)$ is much higher than that of H-beam. The larger central beam density contributes the increase of intensity of the charge exchange line measured, because the line of sight of the viewing array crosses the center of the two beam lines.

The beam density in the plasma is estimated with the Eqs. (2) and (3) based on the density profile measured with Thomson scattering. Figure 5 shows the radial profiles of central beam density (at $w=0$ in Fig. 4) without and with attenuation by plasma $\left(n_{b}^{1}\right)$ and for the full energy compo- 
nent $(j=1)$. Here, beam density is normalized by NBI power of each ion source. Due to the steering and divergence of the neutral beam, the central beam density decreases significantly (by a factor of 2.3 for H-bean and 3.0 for D-beam from $R=4.5 \mathrm{~m}$ to $R=3.0 \mathrm{~m}$ ) even without the attenuation by the plasma. When the beam intensity is attenuated by the plasma, the decrease of the central beam density becomes more pronounced (by a factor of 6 for $\mathrm{H}$ beam and by a factor of 11 for D-beam from $R=4.5 \mathrm{~m}$ to $R=3.0 \mathrm{~m}$ ). These differences in beam density affect the difference in the intensity profile of charge exchange line between H-beam and D-beam as described in the next section. Although the charge exchange spectroscopy provides the local measurements of carbon density, integration effect along the line of sight within the beam width should be taken into account, because the intensity of the charge exchange line is proportional to the line integrated beam density and not to the local beam density at the intersection between line of sight and beam line.

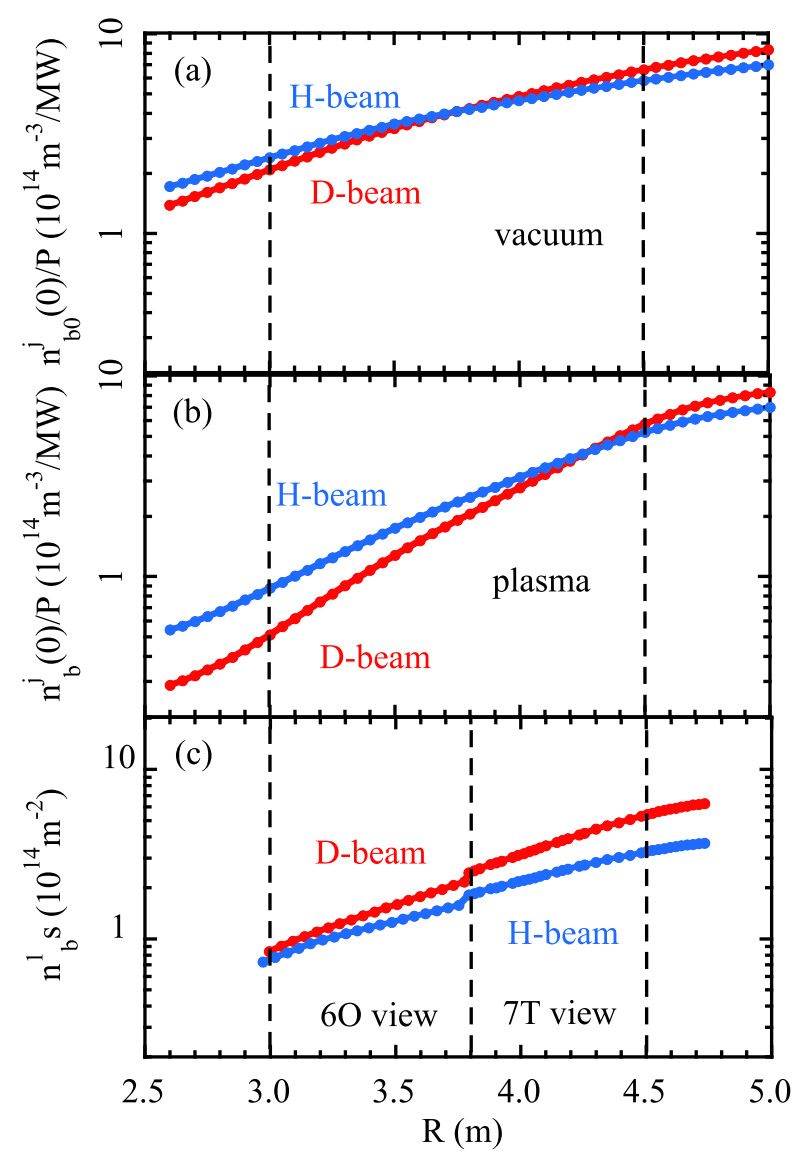

Fig. 5 Radial profiles of central beam density (at $w=0$ in Fig. 4) (a) without attenuation $\left(n_{b 0}^{j}\right)$ and (b) with attenuation by plasma $\left(n_{b}^{1}\right)$ and (c) line integrated beam density $\left(\int n_{b}^{1} d s\right)$ of full energy component of hydrogen and deuterium beam, respectively. Here, beam density is normalized by NBI power of each ion source, while the integrated beam density is the sum of all ion sources with the injected power.
Figure 5 (c) shows the line integrated beam density ( $\int n_{b}^{1} d s$ ) of the full energy component of hydrogen and deuterium beam. Here, the integrated beam density is the total of all ion sources with the injected power. The decrease of line integrated beam density is not so sharp compared with the decrease of central beam density, because the broadening in horizontal direction does not contributes to the decrease of intensity, but only the broadening in vertical direction contributes to the decrease of beam density. The line integrated beam density falls by a factor of 4.2 for H-beam and 6.5 for D-beam from $R=4.5 \mathrm{~m}$ to $R=3.0 \mathrm{~m}$ ). The small jump at $R=3.8 \mathrm{~m}$ is due to the difference in the integration length of between $6 \mathrm{O}$ viewing array and $7 \mathrm{~T}$ viewing array. The 60 view is more perpendicular to the beam line than the $7 \mathrm{~T}$ view and the $6 \mathrm{O}$ view has a slightly shorter integration length than that of the $7 \mathrm{~T}$ view.

\section{Carbon Density Profile Measure- ments Using Hydrogen and Deu- terium Beams}

Once the line integrated beam density is calculated with beam attenuation calculation, the carbon density profile is drawn from the intensity profile of charge exchange line using Eq. (1). In order to eliminate the transport effect, the plasma just after the carbon injection is selected for this study. The carbon density profile is mainly determined by the deposition of carbon pellet rather than the impurity transport in the hydrogen and deuterium plasma.

Figure 6 shows the radial profiles of CVI $(\Delta n=8-7$, $\lambda=529.2 \mathrm{~nm}$ ) line intensity and carbon density for hydrogen and deuterium beam $0.07 \mathrm{~s}$ after the carbon pellet injection. There are differences in the transmission of op-

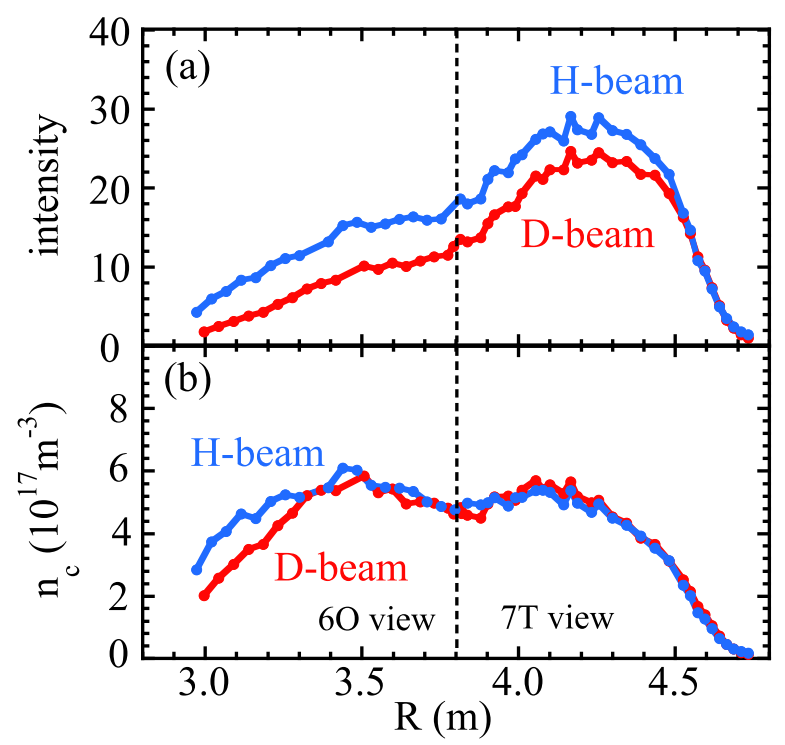

Fig. 6 Radial profiles of (a) CVI $(\Delta n=8-7, \lambda=529.2 \mathrm{~nm})$ line intensity and (b) carbon density for hydrogen and deuterium beam $0.07 \mathrm{~s}$ after the carbon pellet injection. 

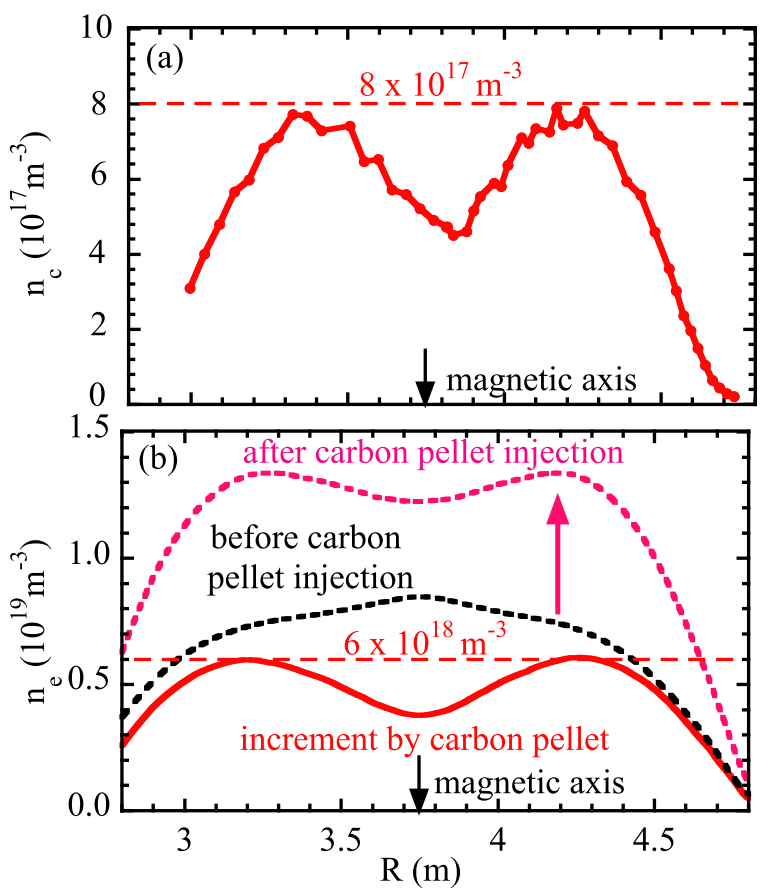

Fig. 7 Radial profile of (a) carbon density $0.05 \mathrm{sec}$ after the carbon pellet injection and (b) electron density $0.01 \mathrm{~s}$ before and $0.05 \mathrm{~s}$ after carbon pellet injection and the difference between these two time slices.

tics between 60 viewing array and $7 \mathrm{~T}$ viewing array. This is because these two arrays have different transmission of the vacuum window and reflection rate of the mirror in the optical path. The transmission and reflection rates of the $6 \mathrm{O}$ view and the $7 \mathrm{~T}$ view also vary during experiment due to the deposition of the plasma. Therefore, the systematic difference in throughput of optical path appears even if the calibration is performed before the LHD is pumped down at the beginning of the experimental campaign. Therefore, the relative calibration of the throughput of these two arrays is performed by adjusting the throughput of the array to derive the same carbon density at the overlapping channel of these two arrays at $R=3.8 \mathrm{~m}$. After the relative calibration, the jump in carbon density profile disappears, although the jump in the CVI intensity profile remains due to the jump of the integration length.

The carbon density profile and the increment of electron density just after the carbon injection $(0.05 \mathrm{~s})$ are compared to check the validity of the carbon density measurements with charge exchange spectroscopy. Figure 7 shows the radial profile of carbon density $0.05 \mathrm{~s}$ after the carbon pellet injection and (b) electron density $0.01 \mathrm{~s}$ before and $0.05 \mathrm{~s}$ after carbon pellet injection and the difference between these two time slices. The carbon density is hollow with the peak of $8 \times 10^{17} \mathrm{~m}^{-3}$ at the half of the plasma minor radius $(R=3.3 \mathrm{~m}$ and $4.2 \mathrm{~m})$ just $0.05 \mathrm{~s}$ after the carbon pellet injection. The increment of electron density of the carbon pellet injection is evaluated from the difference in density profile at two time slices $(0.01 \mathrm{~s}$ before and
$0.05 \mathrm{~s}$ after the carbon pellet injection). The radial profile of the increment of electron density is also hollow at the peak of $6 \times 10^{18} \mathrm{~m}^{-3}$. The hollow profile of the increment of the electron density has a reasonable agreement with the hollow profile of carbon density. The increment of the electron density is consistent with the electron density supplied by the fully ionized carbon $\left(4.8 \times 10^{18} \mathrm{~m}^{-3}\right)$ within the uncertainty of $20 \%$.

\section{Discussion and Summary}

In Fig. 6, the agreement of carbon density profile with $\mathrm{H}$-beam and D-beam is perfect in the region of $R>3.3 \mathrm{~m}$, which is expected for the plasma just after the carbon pellet injection in the similar plasma parameters (magnetic field configuration and strength and plasma density and temperature). However, there is clear difference near the plasma edge at the high magnetic field side $(R<3.3 \mathrm{~m})$. This discrepancy is due to the uncertainty of subtraction of cold component, which is radiated near the plasma edge due to the charge exchange reaction between fully ionized carbon and thermal neutral. The subtraction of the cold component is performed by the neutral beam modulation assuming that the cold component is unchanged between on-time and off-time of the neutral beam, which may not be valid. The change in cold component during the modulation of the neutral beam causes the relatively large uncertainty in the region where the intensity of charge exchange line (hot component) significantly decreases as seen in Fig. 6(a). The effect of halo neutral is significant for the bulk (hydrogen) charge exchange spectroscopy and causes the under estimation of the ion temperature and over estimation of the density of bulk ions [14]. Because of this effect, the discrepancy between hydrogen and carbon ion temperature becomes significant near the plasma center. However, the effect of halo neutral can be neglected for the carbon charge exchange spectroscopy.

In conclusion, a good agreement of carbon density profile after the carbon pellet injection measured with the deuterium beam and with hydrogen beam is a good validation of both the stopping cross section for beam attenuation and emission cross section for the carbon charge exchange line of CVI ( $\Delta n=8-7, \lambda=529.2 \mathrm{~nm})$. Reasonable agreement of profile and magnitude of between the carbon density and increment of electron density confirms the validity of beam attenuation calculations and atomic data used in these calculations.

\section{Acknowledgments}

The authors are grateful to the technical staff of LHD for their excellent support for this work.This work is supported by the National Institute for Fusion Science grant administrative budgets (NIFS10ULHH021, NIFS17KLPH030) and JSPS KAKENHI Grant Numbers JP15H02336, JP16H02442, JP17H01368. 
[1] K. Kim, et al., Phys. Plasmas 24, 062302 (2017).

[2] N. Bonanomi, et al., Nucl. Fusion 58, 026028 (2018).

[3] N. Bonanomi, et al., Nucl. Fusion 58, 036009 (2018).

[4] H. Takahashi, et al., Nucl. Fusion 58, 106028 (2018).

[5] K. Mukai, et al., Plasma Phys. Control Fusion 60, 074005 (2018).

[6] K. Ida, et al., Nucl. Fusion 59 (2019) to be published.

[7] R.J. Fonck, et al., Phys. Rev. A 29, 3288 (1984).
[8] K. Ida, et al., Phys. Sci. Instrum. 60, 867 (1989).

[9] K. Ida, et al., Phys. Sci. Instrum. 71, 2360 (2000).

[10] M. Yoshinuma, et al., Fusion Sci. Technol. 58, 375 (2010).

[11] H.P. Summers, The ADAS User Manual version 2.6 www.adas.ac.uk (2004).

[12] W.M. Solomon, et al., Phys. Sci. Instrum. 75, 3481 (2004).

[13] J. Chen, et al., Phys. Lett. A 383, 1293 (2019).

[14] K. Ida, et al., Phys. Sci. Instrum. 86, 123514 (2015). 